\title{
Association between shift work and obesity among female nurses: Korean Nurses' Survey
}

\author{
Min-Ju Kim ${ }^{1}$, Kuk-Hui Son ${ }^{1}$, Hyun-Young Park ${ }^{1 *}$, Dong-Ju Choi ${ }^{2}$, Chang-Hwan Yoon², Hea-Young Lee ${ }^{3}$, \\ Eun-Young Cho ${ }^{4}$ and Myeong-Chan Cho ${ }^{5,6}$
}

\begin{abstract}
Background: Shift work has been hypothesized as a risk factor for obesity. In this study, we investigated the association between current shift work and body mass index (BMI) among female nurses in Korea. The relationship between duration of shift work and BMl of the participants was also evaluated.

Methods: This cross-sectional survey evaluated participants in the Korean Nurses' Survey, conducted from October to December 2011, using web-based self-administered questionnaires. A total of 9,989 nurses were included among 10,000 who registered on the survey web site $(5,287$ shift workers and 4,702 non-shift workers). Current shift workers were divided into tertiles of shift work duration ( $0.08-3.00$ years, $n=1,732 ; 3.08-6.75$ years, $n=1,731$; and $6.83-38.00$ years, $n=1,686$ ). The BMl thresholds of overweight and obesity were $\geq 23 \mathrm{~kg} / \mathrm{m}^{2}$ and $\geq 25 \mathrm{~kg} / \mathrm{m}^{2}$, respectively. Data were analyzed using SPSS software.

Results: Mean participant age was $33.2 \pm 8.6$ years and the mean BMI was $20.9 \pm 2.5 \mathrm{~kg} / \mathrm{m}^{2}$. There were statistically significant differences in current smoking status, regular drinking habit, dietary habits, regular exercise, sleep problems and self-perceived health status according to duration of shift work. The overall prevalence of overweight/obesity (18.6\%) and obesity (7.4\%) increased significantly as shift work duration increased from the lowest to highest tertile $(P$ for trend $<0.001$ ). Multivariate logistic regression analysis revealed no association between current shift work and BMI. However, after adjusting for potential confounders, the participants with the longest duration of shift work were $1.63(95 \% \mathrm{Cl}, 1.22-2.17)$ times more likely to be overweight or obese than those with the shortest duration. There was a significant positive association between obesity and shift work duration in the unadjusted analysis; however, it was attenuated and no longer significant in the multivariate model.

Conclusions: The duration of shift work was positively associated with prevalence of overweight/obesity in nurses in Korea. Although these findings need to be confirmed in prospective studies, they suggest that special attention should be paid to female nurses with a long duration of shift work.
\end{abstract}

Keywords: Shift work, Body mass index, Overweight, Obesity

\section{Background}

Shift work is defined as work outside of daytime hours, including irregular or rotating schedules, and evening and night work [1]. Shift work has been associated with gastrointestinal disease, cardiovascular disease, diabetes, several types of cancer, and metabolic disorders [1-3]. The mechanisms linking shift work to health problems are not clear, but changed circadian rhythm, sleep problems, stress, and

\footnotetext{
* Correspondence: mdhypark@gmail.com

'Division of Cardiovascular and Rare Diseases, Center for Biomedical Science, National Institute of Health, Chungbuk, Korea

Full list of author information is available at the end of the article
}

lifestyle and behavioral changes such as diet and smoking might be potential mediators [3]. Weight gain in shift workers may be related to several mechanisms which involved alternations of genetic factors, glucose and lipid homeostasis, thermogenic response due to a night eating pattern and neurohumoral factors such as leptin and ghrelin [4].

Obesity is a risk factor for chronic diseases, and a number of studies report that overweight and obesity are more prevalent among shift workers than day workers [5-7]. In addition, several studies show that there are significant correlations between shift work and weight gain, overweight

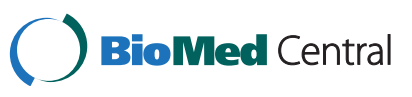


or obesity [8]. Because shift work is an occupational characteristic of nurses, working schedules may influence nurses' health by increasing the risk for weight gain and obesity [9]. Two studies in nurses working in the United States found that the combined prevalence of overweight and obesity was $55.5 \%$ to $65.4 \%[10,11]$, similar to or slightly lower than the age-adjusted prevalence among the US general population, which is $63.7 \%$ [12].

Shift work is considered a risk factor for obesity, and studies examining this relationship have been conducted in various occupational groups in a number of different countries [8]. However, only a few studies with limited data have reported the impact of occupational characteristics on the body mass index (BMI) of female nurses [8]. In addition, few studies have examined the duration of shift work in relation to obesity [13-15]. Previous founding suggested that the risk of overweight and/or obesity for shift work may increase by at least 39\% [15-17]. Therefore, in this study, we investigated the association between current shift work and BMI among female nurses in Korea. The relationship between duration of shift work and the BMI of participants was also evaluated.

\section{Methods}

\section{Study participants}

The Korean Nurses' Survey is a cross-sectional survey conducted for the purpose of evaluating the association between lifestyle and Korean women's health. The Korea National Institute of Health collaborated with Korean Nurses Association and Seoul National University's Bundang Hospital to perform this survey. The Korean Nurses Association sent e-mails containing a direct link to access a web site with an introduction of the survey, questionnaires and an informed consent form. A total of 10,000 nurses registered on the website from October to December 2011 and completed the web-based selfadministered questionnaires. Six subjects who were younger than 20 years of age, one who was not employed in nursing, and four with incomplete data on working years and BMI were excluded from the study. A total of 9,989 nurses were included. The nurses were divided into two groups according to current shift work, leaving 5,287 shift workers and 4,702 non-shift workers. Nurses not currently performing shift work were excluded from the main analyses. Among the 5,287 current shift workers, 138 with missing data on shift work duration were excluded; 5,149 were selected for further analysis. The remaining subjects were divided into tertile of shift work duration: Tertile 1, 0.08-3.00 years, $\mathrm{n}=1,732$; Tertile 2, 3.086.75 years, $n=1,731$; and Tertile $3,6.83-38.00$ years, $\mathrm{n}=1,686$. The study protocol was approved by the Institutional Review Board of Seoul National University's Bundang Hospital. Informed consent was obtained from all study participants.

\section{Measurements}

The questionnaires collected information on demographic characteristics, comorbidities, cardiovascular disease symptoms, medical history, family history of diseases, reproductive health, lifestyle, psychiatric and occupational characteristics, dietary patterns and food intake.

BMI was calculated as body weight $(\mathrm{kg})$ divided by height squared $\left(\mathrm{m}^{2}\right)$. The BMI thresholds were $\geq 23 \mathrm{~kg} / \mathrm{m}^{2}$ for overweight and $\geq 25 \mathrm{~kg} / \mathrm{m}^{2}$ for obesity. Participants were considered as shift workers if they answered 'yes' to the question 'Do you currently work in shifts?', and then provided information on shift work duration. Smoking status was characterized as 'never', 'past', and 'current'. Current smokers were defined as those who had smoked more than 100 cigarettes in their lifetime and also reported that they were 'currently smoking'. Participants were considered regular drinkers if they reported consuming alcohol more than once a month. Dietary habits were estimated using the question 'Do you eat breakfast, lunch and dinner?' Respondents who answered 'never' to eating any of the three meals were characterized as 'meal skipping'. Subjects were assigned to the regular exercise group if they performed vigorous or moderateintensity physical activity that caused substantial or slight increase in breathing or heart rate more than once a week. Sleep problems were evaluated by asking about the frequency of difficulty in sleeping or excessive sleep in the past 2 weeks. The four possible responses were: never, 1-6 days, 7-13 days, and every day. Self-perceived health status was determined by asking 'how you rate your state of health in general', for which the five responses were: very good, good, fair, so-so, and bad.

\section{Statistical analysis}

Baseline characteristics of the study participants were summarized according to current shift work, and were presented by tertile of shift work duration. Continuous variables were expressed as mean \pm standard deviations (SD) and categorical variables as percentages. Statistically significant differences between groups were calculated by the independent $t$-test and one-way analysis of variance (ANOVA) with Duncan's multiple comparison test. The chi-square test was used to evaluate differences between groups for categorical variables. A linear-by-linear association test was performed to assess trends of overweight and obesity prevalence according to shift work duration. Multivariate logistic regression analysis was conducted to identify associations of shift work with overweight and obesity. Odds ratios (ORs) and 95\% confidence intervals (CIs) were calculated. Potential confounding variables that were adjusted for were age (continuous), current smoking status (yes/no), regular drinking habit (yes/no), breakfast skipping (yes/no), regular exercise (yes/no), marital status (married/cohabitation/divorced/widowed/never- 
married.), family income (less than 30 million won/3045 million won/45-75 million won/more than 75 million won), education (graduation from 3-year course/graduation from 4-year course/graduate school or higher), sleep problems (never or 1-6 days/7-13 days or every day) and selfperceived health status (very good or good/fair, so-so or bad). Relationships of age, BMI and shift work duration were examined with the Pearson correlation. Spearman correlation coefficients between shift work duration and lifestyle factors were calculated. Adjusted ORs for overweight/obesity and obesity by selected factors were also calculated in a logistic regression model. Values of $P<0.05$ were considered to be statistically significant. All data were analyzed using SPSS Statistics 21 (SPSS Inc., IBM Corp., Chicago, IL, USA).

\section{Results}

Baseline characteristics

Baseline characteristics of the study participants according to current shift work are shown in Table 1. The mean age was $33.2 \pm 8.6$ years and their mean BMI was $20.9 \pm 2.5 \mathrm{~kg} / \mathrm{m}^{2}$. Non-shift workers had a higher mean BMI than current shift workers $\left(21.4 \pm 2.6 \mathrm{~kg} / \mathrm{m}^{2} \mathrm{vs}\right.$. $\left.20.5 \pm 2.5 \mathrm{~kg} / \mathrm{m}^{2}, P<0.001\right)$. Of all subjects, the prevalence of current smokers was relatively low (1.2\%) and almost half of the participants were regular drinkers. About $36.5 \%$ of subjects skipped breakfast, while $1.3 \%$ and $1.0 \%$ skipped lunch and dinner, respectively. The prevalence of skipping breakfast was higher in shift workers compared to non-shift workers (43.1\% vs. $29.2 \%)$. Current shift workers were less likely to exercise regularly than non-shift workers (41.2\% vs. $47.3 \%)$. Sleep problems for 7-13 days or every day during the past 2 weeks were more frequent among shift workers (27.2\%). There were statistically significant differences in self-perceived health status according to current shift work $(P<0.001)$. For further analysis, current shift workers were organized according to tertile of shift work duration. Among the current shift workers, subjects with the longer duration of shift work were more likely to be older $(P<0.001)$ and to have a higher BMI $(P<0.001)$. Those subjects were also more likely to have hypertension, diabetes, hyperlipidemia or breast cancer $(P=0.003, P<0.001$, $P=0.003, P<0.001)$. The prevalence of regular exercise was the highest in Tertile 2 (44.7\%), followed by Tertile 3 (40.1\%) and Tertile 1 (38.6\%). Difficulty with sleeping or excessive sleep for 7-13 days or every day was most frequent in Tertile 1 (32.9\%), followed by Tertile 2 (30.9\%) and Tertile 3 (17.8\%).

\section{Prevalence of overweight and obesity}

Table 2 shows the prevalence of overweight and obesity according to current shift work and shift work duration. The overall prevalence of overweight or obesity and obesity was $18.6 \%$ and $7.4 \%$, respectively. Non-shift workers had a higher prevalence of overweight and obesity than shift workers $(P<0.001)$. The prevalence increased significantly as shift work duration increased from Tertile 1 to Tertile 3 ( $P$ for trend $<0.001)$.

\section{Results of multivariate logistic regression analysis of overweight and obesity}

The results of multivariate logistic regression analysis of overweight and obesity according to current shift work are presented in Additional file 1. The ORs of overweight/ obesity and obesity in unadjusted analysis were 0.51 (95\% CI, 0.46-0.57) and 0.62 (95\% CI, 0.53-0.72), respectively. However, no association was found in any of the adjusted models.

When the non-shift work group was used as a reference, Tertile 3 of shift work duration had a significantly increased risk for overweight/obesity (OR, 1.24; 95\% CI, 1.07-1.43) and obesity (OR, 1.32; 95\% CI, 1.08-1.62) after fully adjusting for covariates, including age [see Additional file 2]. Unadjusted, age-adjusted, and multivariate-adjusted ORs for overweight and obesity according to duration of shift work are shown in Table 3 . When the overweight and obese participants were combined into one group in the unadjusted analysis, the ORs of overweight/obesity in Tertile 3 of shift work duration was 3.40 (95\% CI, 2.774.19) compared with Tertile $1(P<0.001)$. After adjusting for potential confounding variables in multivariate logistic regression analysis, the participants in Tertile 3 were 1.63 (95\% CI, 1.22-2.17) times more likely to be overweight/ obese than those in Tertile $1(P=0.001)$. Similar results were also observed in overweight subjects. The participants in Tertile 3 were significantly associated with the increased risk of overweight (data not shown). There were significant, positive correlations between obesity and shift work duration in the unadjusted analysis. Although the association was statistically not significant after adjustment for age, statistically significant correlations were observed after adjustment for current smoking status, regular drinking habit, breakfast skipping and regular exercise $(P=0.028)$. However, no association was observed in the multivariate model. Tertile 2 showed the increased trends for an increased risk of overweight/ obesity or obesity in the adjusted models, although this association was not statistically significant. Age, current smoking status, marital status, family income, education, breakfast skipping, regular exercise and sleep problems were each significantly associated with overweight/ obesity when included as covariates in the logistic regression models [see Additional file 3]. The factors that were significantly associated with obesity included age, current smoking status, marital status, breakfast skipping, regular exercise and self-perceived health status [see Additional file 4]. 
Table 1 Mean values of baseline characteristics according to current shift work and tertile of shift work duration

\begin{tabular}{|c|c|c|c|c|c|c|c|c|}
\hline \multirow[t]{2}{*}{ Variables } & \multirow{2}{*}{$\begin{array}{c}\text { Total } \\
(n=9,989)\end{array}$} & \multicolumn{2}{|c|}{ Current shift work } & \multirow[t]{2}{*}{$P$-value } & \multicolumn{3}{|c|}{ Tertile of shift work duration } & \multirow[t]{2}{*}{$P$-value } \\
\hline & & $\begin{array}{c}\text { No } \\
(n=4,702)\end{array}$ & $\begin{array}{c}\text { Yes } \\
(n=5,287)\end{array}$ & & $\begin{array}{c}\text { Tertile } 1 \\
(\mathrm{n}=1,732)\end{array}$ & $\begin{array}{c}\text { Tertile } 2 \\
(n=1,731)\end{array}$ & $\begin{array}{c}\text { Tertile } 3 \\
(n=1,686)\end{array}$ & \\
\hline Age (years) & $33.2 \pm 8.6$ & $37.6 \pm 9.2$ & $29.2 \pm 5.6$ & $<0.001$ & $24.9 \pm 2.9^{c}$ & $28.1 \pm 3.1^{b}$ & $34.7 \pm 5.1^{a}$ & $<0.001$ \\
\hline BMI $\left(\mathrm{kg} / \mathrm{m}^{2}\right)$ & $20.9 \pm 2.5$ & $21.4 \pm 2.6$ & $20.5 \pm 2.5$ & $<0.001$ & $20.0 \pm 2.1^{c}$ & $20.2 \pm 2.4^{b}$ & $21.3 \pm 2.7^{\mathrm{a}}$ & $<0.001$ \\
\hline Hypertension & $220(2.2)$ & $193(4.1)$ & $27(0.5)$ & $<0.001$ & $3(0.2)$ & $6(0.3)$ & $16(0.9)$ & 0.003 \\
\hline Diabetes & $43(0.4)$ & $31(0.7)$ & $12(0.2)$ & 0.001 & $3(0.2)$ & $4(0.2)$ & $5(0.3)$ & $<0.001$ \\
\hline Osteoporosis & $102(1.0)$ & $79(1.7)$ & $23(0.4)$ & $<0.001$ & $1(0.1)$ & $6(0.3)$ & $13(0.8)$ & 0.003 \\
\hline Hyperlipidemia & $57(7.6)$ & $472(10.0)$ & $285(5.4)$ & $<0.001$ & $40(2.3)$ & $78(4.5)$ & $165(9.8)$ & $<0.001$ \\
\hline Breast cancer & $87(0.9)$ & $73(1.6)$ & $14(0.3)$ & $<0.001$ & $1(0.1)$ & $3(0.2)$ & $10(0.6)$ & 0.007 \\
\hline Current smoking status & & & & 0.335 & & & & $<0.001$ \\
\hline No & $9874(98.8)$ & $4653(99.0)$ & $5221(98.8)$ & & $1697(98.0)$ & $1718(99.2)$ & 1675 (99.3) & \\
\hline Yes & $115(1.2)$ & $49(1.0)$ & $66(1.2)$ & & $35(2.0)$ & $13(0.8)$ & $11(0.7)$ & \\
\hline Regular drinking habit & & & & $<0.001$ & & & & $<0.001$ \\
\hline No & $5144(51.5)$ & $2683(57.1)$ & $2461(46.5)$ & & $625(36.1)$ & $788(45.5)$ & $993(58.9)$ & \\
\hline Yes & $4845(48.5)$ & 2019 (42.9) & $2826(53.5)$ & & $1107(63.9)$ & $943(54.5)$ & $693(41.1)$ & \\
\hline Marital status & & & & $<0.001$ & & & & $<0.001$ \\
\hline Married (remarriage) & $4589(45.9)$ & $3087(65.7)$ & $1502(28.4)$ & & $88(5.1)$ & $344(19.9)$ & $1032(61.2)$ & \\
\hline Cohabitation & $16(0.2)$ & $6(0.1)$ & $10(0.2)$ & & $3(0.2)$ & $2(0.1)$ & $4(0.2)$ & \\
\hline Divorced & $246(2.5)$ & $127(2.7)$ & $119(2.3)$ & & $31(1.8)$ & $42(2.5)$ & $40(2.4)$ & \\
\hline Widowed & $36(0.4)$ & $34(0.7)$ & $2(0.0)$ & & $0(0.0)$ & $0(0.0)$ & $2(0.1)$ & \\
\hline Never-married & $5102(51.1)$ & $1448(30.8)$ & $3654(69.1)$ & & $1610(93.0)$ & $1341(77.5)$ & $608(36.1)$ & \\
\hline Family income & & & & $<0.001$ & & & & $<0.001$ \\
\hline Less than 30 million won & $1259(12.6)$ & $490(10.4)$ & $769(14.5)$ & & $401(23.2)$ & $247(14.3)$ & $96(5.7)$ & \\
\hline 30-45 million won & $2787(27.9)$ & $1002(21.3)$ & $1785(33.8)$ & & $605(34.9)$ & $685(39.6)$ & $454(26.9)$ & \\
\hline 45-75 million won & $3545(35.5)$ & $1802(38.3)$ & $1743(33.0)$ & & $472(27.3)$ & $522(30.2)$ & $699(41.5)$ & \\
\hline More than 75 million won & $2389(24.0)$ & 1408 (29.9) & $990(18.7)$ & & $254(14.7)$ & $277(16.0)$ & $437(25.9)$ & \\
\hline Education & & & & $<0.001$ & & & & $<0.001$ \\
\hline Graduation from three-year course & $3469(34.7)$ & $1294(27.5)$ & $2175(41.1)$ & & $845(48.8)$ & $733(42.3)$ & $528(31.3)$ & \\
\hline Graduation from four-year course & $4839(48.4)$ & $2083(44.3)$ & $2756(52.1)$ & & $880(50.8)$ & $954(55.1)$ & $862(51.1)$ & \\
\hline Graduate school or higher & $1681(16.8)$ & $1325(28.2)$ & $356(6.7)$ & & $7(0.4)$ & $44(2.5)$ & $296(17.6)$ & \\
\hline \multicolumn{9}{|l|}{ Dietary habits } \\
\hline Breakfast skipping & $3649(36.5)$ & $1371(29.2)$ & $2278(43.1)$ & $<0.001$ & $749(43.2)$ & $815(47.1)$ & $647(38.4)$ & $<0.001$ \\
\hline Lunch skipping & $129(1.3)$ & $23(0.5)$ & $106(2.0)$ & $<0.001$ & $50(2.9)$ & $35(2.0)$ & $19(1.1)$ & $<0.001$ \\
\hline Dinner skipping & $96(1.0)$ & $38(0.8)$ & $58(1.1)$ & 0.140 & $20(1.2)$ & $23(1.3)$ & $14(0.8)$ & $<0.001$ \\
\hline Regular exercise & & & & $<0.001$ & & & & $<0.001$ \\
\hline No & $5588(55.9)$ & $2478(52.7)$ & $3110(58.8)$ & & $1063(61.4)$ & $957(55.3)$ & $1010(59.9)$ & \\
\hline Yes & $4401(44.1)$ & $2224(47.3)$ & $2177(41.2)$ & & $669(38.6)$ & $774(44.7)$ & $676(40.1)$ & \\
\hline Sleep problems, during the last 2 weeks & & & & $<0.001$ & & & & $<0.001$ \\
\hline Never & $4540(45.4)$ & $2815(59.9)$ & $1725(32.6)$ & & $477(27.5)$ & $494(28.5)$ & $707(41.9)$ & \\
\hline 1-6 days & $3538(35.4)$ & $1414(30.1)$ & $2124(40.2)$ & & $685(39.5)$ & $702(40.6)$ & $679(40.3)$ & \\
\hline 7-13 days & $1187(11.9)$ & $304(6.5)$ & $883(16.7)$ & & $333(19.2)$ & $331(19.1)$ & $202(12.0)$ & \\
\hline Every day & $724(7.2)$ & 169 (3.6) & $555(10.5)$ & & $237(13.7)$ & $204(11.8)$ & $98(5.8)$ & \\
\hline Self-perceived health status & & & & $<0.001$ & & & & 0.003 \\
\hline Very good & $716(7.2)$ & $389(8.3)$ & $327(6.2)$ & & $119(6.9)$ & $105(6.1)$ & $92(5.5)$ & \\
\hline Good & 4545 (45.5) & $2219(47.2)$ & $2326(44.0)$ & & 804 (46.4) & 756 (43.7) & $710(42.1)$ & \\
\hline
\end{tabular}


Table 1 Mean values of baseline characteristics according to current shift work and tertile of shift work duration (Continued)

\begin{tabular}{lcccccc}
\hline Fair & $3837(38.4)$ & $1735(36.9)$ & $2102(39.8)$ & $662(38.2)$ & $666(38.5)$ & $716(42.5)$ \\
So-so & $753(7.5)$ & $290(6.2)$ & $463(8.8)$ & $134(7.7)$ & $174(10.1)$ & $143(8.5)$ \\
Bad & $138(1.4)$ & $69(1.5)$ & $69(1.3)$ & $13(0.8)$ & $30(1.7)$ & $25(1.5)$ \\
\hline
\end{tabular}

Data are expressed as mean \pm SD or percentage. Statistical differences between groups were compared with independent sample $t$-test and one-way ANOVA for continuous variables and chi-square test for categorical variables.

$a, b, c$ The same letters indicate non-significant difference between groups based on Duncan's multiple comparison test.

\section{Discussion}

The purpose of this study was to examine the relationship between shift work and BMI among nurses in Korea. The prevalence of overweight/obesity and obesity significantly increased with increasing shift work duration. Although there was no association between current shift work and BMI, we found that the subjects in the highest tertile of shift work duration were more likely to be overweight/ obese after adjustment for potential confounders.

In this sample of 9,989 female nurses, $66.5 \%$ were of normal weight, suggesting that overweight or obesity is not common in the population of nurses in Korea. In addition, $18.6 \%$ of the participants were either overweight $(11.2 \%)$ or obese $(7.4 \%)$, which is lower than the agestandardized obesity prevalence of $25.7 \%$ in the general Korean population, as reported by the Korean National Health and Nutrition Examination Survey (KNHANES) [18]. These results might be explained either by a healthyworker effect among nurses or the large proportion of participants who were $20-29$ years of age $(44.4 \%)$ and $30-39$ years of age (33.8\%). The BMI in non-shift workers was higher than that in current shift workers, which might have been influenced by the relatively older age of nonshift workers, as well as other potential confounding factors. When adjusted for potential confounders in multivariate logistic regression analysis, no significant association was found between current shift work and BMI. Because non-shift workers had not provided any information about past shift work experience, they were excluded from the main analyses.

In a further analysis, we also found that most of the subjects in the lowest tertile of shift work duration had never married (93.0\%), whereas those in the highest tertile were more likely to be married (61.2\%). As shown in Additional files 3 and 4, married women who were current shift workers had a higher risk of overweight/ obesity and obesity compared to never-married women, which is consistent with an earlier study [16]. In the present study, the finding that nurses with lower levels of education and income had an increased risk of overweight/obesity could be explained by a decreased interest in health problems in this group.

Several studies report finding associations between shift work and weight gain, overweight or obesity [14-16,19]. The female workers in electronic device factories in Malaysia who worked night shifts had significantly elevated ORs for being overweight, even after adjustments [16]. A study including 377 shift workers and non-shift workers found a positive relationship between duration of shift work and BMI [14], and a cross-sectional survey found that longer exposure to shift work was a highly significant predictor of increased BMI [15]. Our results were consistent with those obtained in earlier studies; multiple logistic regression revealed that Tertile 3 of shift work duration had a significantly increased risk for overweight/obesity (OR, 1.24; 95\% CI, 1.07-1.43) and obesity

Table 2 Prevalence of overweight/obesity and obesity according to current shift work and tertile of shift work duration

\begin{tabular}{|c|c|c|c|c|c|c|c|c|c|c|c|c|c|c|}
\hline \multirow[t]{2}{*}{ Variables } & \multicolumn{2}{|c|}{ Total } & \multicolumn{4}{|c|}{ Current shift work } & \multirow[t]{2}{*}{$P$-value } & \multicolumn{6}{|c|}{ Tertile of shift work duration } & \multirow[t]{2}{*}{$P$ for trend } \\
\hline & \multicolumn{2}{|c|}{$(n=9,989)$} & \multicolumn{2}{|c|}{$\begin{array}{c}\text { No } \\
(n=4,702)\end{array}$} & \multicolumn{2}{|c|}{$\begin{array}{c}\text { Yes } \\
(n=5,287)\end{array}$} & & \multicolumn{2}{|c|}{$\begin{array}{c}\text { Tertile } 1 \\
(\mathrm{n}=1,732)\end{array}$} & \multicolumn{2}{|c|}{$\begin{array}{c}\text { Tertile } 2 \\
(n=1,731)\end{array}$} & \multicolumn{2}{|c|}{$\begin{array}{c}\text { Tertile } 3 \\
(n=1,686)\end{array}$} & \\
\hline \multicolumn{15}{|l|}{ BMI category } \\
\hline$<23 \mathrm{~kg} / \mathrm{m}^{2}$ & 8134 & $(81.4)$ & 3578 & $(76.1)$ & 4556 & $(86.2)$ & $<0.001$ & 1593 & $(92.0)$ & 1546 & $(89.3)$ & 1300 & $(77.1)$ & $<0.001$ \\
\hline$\geq 23 \mathrm{~kg} / \mathrm{m}^{2} \mathrm{a}$ & 1855 & (18.6) & 1124 & $(23.9)$ & 731 & (13.8) & & 139 & $(8.0)$ & 185 & $(10.7)$ & 386 & $(22.9)$ & \\
\hline \multicolumn{15}{|l|}{ BMI category } \\
\hline$<25 \mathrm{~kg} / \mathrm{m}^{2}$ & 9253 & $(92.6)$ & 4274 & $(90.9)$ & 4979 & $(94.2)$ & $<0.001$ & 1678 & $(96.9)$ & 1647 & $(95.1)$ & 1524 & $(90.4)$ & $<0.001$ \\
\hline$\geq 25 \mathrm{~kg} / \mathrm{m}^{2 \mathrm{~b}}$ & 736 & $(7.4)$ & 428 & $(9.1)$ & 308 & $(5.8)$ & & 54 & (3.1) & 84 & $(4.9)$ & 162 & $(9.6)$ & \\
\hline
\end{tabular}

Data are expressed as $\mathrm{n}(\%)$ and tested by chi-square test.

${ }^{a}$ Overweight and obesity combined.

${ }^{\mathrm{b}}$ Obesity. 
Table 3 Multivariate-adjusted odds ratios (ORs) for overweight/obesity and obesity according to tertile of shift work duration

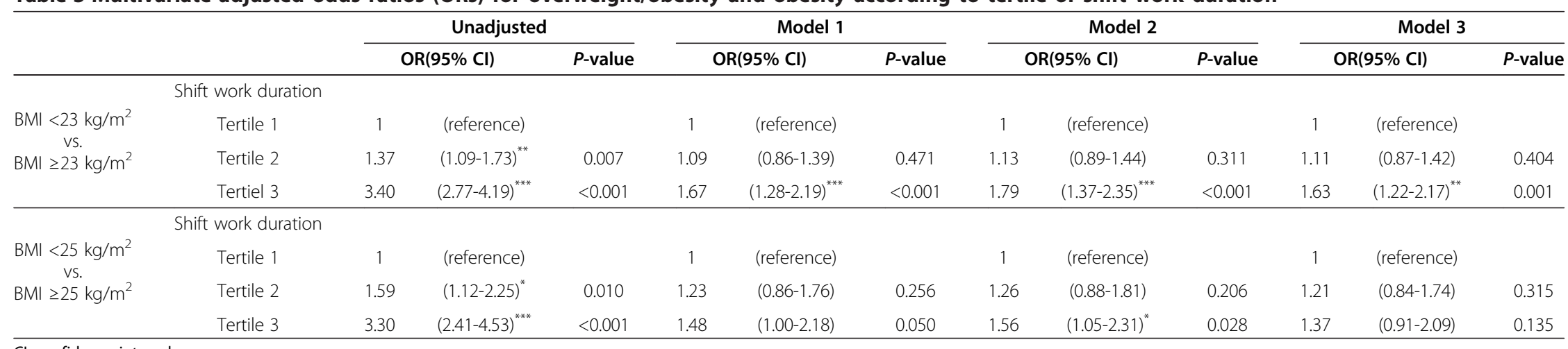

$\mathrm{Cl}$, confidence interval.

Model 1: adjusted for age

Model 3: adjusted for age, current smoking status, regular drinking habit, breakfast skipping, regular exercise, marital status, family income, education, sleep problem and self-perceived health status.

${ }^{*} \mathrm{P}<0.05,{ }^{* * *} \mathrm{P}<0.01,{ }^{* * * *} \mathrm{P}<0.001$ 
(OR, 1.32; 95\% CI, 1.08-1.62) compared with non-shift work group. When the shortest duration of shift work was used as a reference, the nurses with the longest duration of shift work had a 1.63 times greater risk of overweight/obesity. Similarly, a cross-sectional study from Australia found that nurses working shifts were 1.15 times more likely to be overweight or obese than day workers, and nightonly shift work was associated with obesity only [20,21]. A study in 85 hospital shift workers reported that mean weight gain was greater in those on evening and night shifts $(4.3 \mathrm{~kg})$ than in those on day shifts $(0.9 \mathrm{~kg})$ [13]. Although our findings showed no association between shift work duration and obesity in the multivariate model, statistically significant correlations was observed after adjustment for age, current smoking status, regular drinking habit, breakfast skipping and regular exercise. This might be explained by the relatively low proportion of obese nurses among current shift workers (5.8\% total; $3.1 \%$ in Tertile $1,4.9 \%$ in Tertile 2 , and $9.6 \%$ in Tertile 3 ). Therefore, we combined overweight and obesity into one category as dependent variables. For further analysis, we selected the participants over the age of 30 years. Tertile 2 and Tertile 3 showed the significant association with overweight/obesity after adjustment for potential confounders (OR, 1.45; 95\% CI, 1.07-1.98 and OR, 1.45; $95 \% \mathrm{CI}, 1.04-2.02)$. There was a significant association between obesity and Tertile 3 in the unadjusted analysis (OR, 1.59; 95\% CI, 1.07-2.36); however, it was no longer significant in the adjusted models.

It is well known that lifestyle factors are potential mediators linking shift work to BMI [3]. In a logistic regression model including confounding factors as covariates, the factors that remained significantly associated with overweight/obesity were age, current smoking status, marital status, family income, education, breakfast skipping and sleep problems. It is known that inadequate sleep and poor quality sleep are associated with obesity [7]. Consistent with those findings, our results showed that sleep problems were associated with overweight/obesity [see Additional file 3]. Approximately 27\% of shift workers responded that they had difficulty with sleeping or excessive sleep for 7-13 days or every day during the past 2 weeks. Interestingly, sleep problems were reported most frequently in Tertile 1 (32.9\%), followed by Tertile 2 (30.9\%) and Tertile 3 (17.8\%), showing a higher prevalence among subjects who started shift work recently. One previous study reported that nurses' diet and exercise habits were negatively influenced by sleep disturbance by working at night [22], and that they also faced changes in eating habits and food selection. Previous studies found that midnight shift workers had the highest total energy intake and usually consumed more calories in the evening [4]. In another study, $40 \%$ of nurses who recognized that they were overweight or obese responded that they ate a healthy diet and exercise regularly, but could not lose excess weight [23]. In the present study, $43.1 \%$ of shift workers skipped breakfast. The percentage was highest in Tertile $2(47.1 \%)$, followed by Tertile 1 (43.2\%), and Tertile 3 (38.4\%). In addition, there was an inverse relationship with breakfast skipping and the risk of overweight and obesity. In the current study, we did not investigate diet quality, types, or amount of food eaten and energy intake; this should be further examined. In general, physical activity is inversely associated with obesity; however, our results indicated that regular exercise had a positive relationship with overweight/obesity. The prevalence of shift workers who exercised regularly was $40.3 \%$ in those with BMI $<23 \mathrm{~kg} / \mathrm{m}^{2}$ and $46.2 \%$ in those with $\mathrm{BMI} \geq 23 \mathrm{~kg} / \mathrm{m}^{2}$. This might imply that nurses who recognized that they were overweight or obese were more likely to exercise regularly. Because this was a crosssectional study, additional investigations are needed for better understanding of the relationship between shift work and physical activity. Interestingly, the participants with the longest duration of shift work had a significantly increased risk of hyperlipidemia after adjustment for age (OR, 1.68; 95\% CI, 1.11-2.55).

This study included a large, representative sample of nurses in Korea and it confirmed an association between increasing duration of shift work and overweight/obesity after considering obesity-related lifestyle factors as confounders. However, there are some study limitations. First, as the study was cross-sectional in design, it is difficult to conclude that the relationship between shift work and BMI was causal. Further longitudinal study will be needed to achieve higher levels of evidence. Second, our data were collected from self-reported questionnaires. Additional metabolic markers should be included in future research for a better understanding of the relationship between shift work and body weight. Third, nurses not currently performing shift work might be misclassified because they had not provided any information about past shift work experience. Finally, shift work exposures at multiple aspects, such as frequency of night shifts, duration of each shifts, speed and direction of shift rotation, were not collected in the current study. Accordingly, further studies will be needed to assess the relationships of different kinds of schedules and duration of shift work to obesity.

\section{Conclusions}

In conclusion, we found that increased duration of shift work was associated with higher risk of overweight/obesity in shift-working nurses in Korea. Although our findings need to be confirmed in prospective studies, they suggest that special attention should be paid to female nurses with long durations of shift work. It would also be valuable to investigate which shift schedules are related to obesity. 


\section{Additional files}

Additional file 1: Multivariate-adjusted odds ratios (ORs) for overweight/obesity and obesity according to current shift work.

Additional file 2: Multivariate-adjusted odds ratios (ORs) for overweight/obesity and obesity according to shift work duration.

Additional file 3: Adjusted odds ratios (ORs) for overweight/obesity by selected factors among current shift workers.

Additional file 4: Adjusted odds ratios (ORs) for obesity by selected factors among current shift workers.

\section{Abbreviations}

BMI: Body mass index; SD: Standard deviations; ANOVA: One-way analysis of variance; ORs: Odds ratios; Cls: Confidence intervals; KNHANES: Korean National Health and Nutrition Examination Survey.

\section{Competing interests}

The authors declare that they have no competing interests.

\section{Authors' contributions}

MJK performed the statistical analysis and participated in drafting the manuscript. KHS contributed data collection and management. HYP participated in the design of the study, revised the manuscript for important intellectual content, and gave final approval of the version to be published. DJC is the principal investigator of this study, and made substantial contributions to study design and discussion. CHY and HYL collected data, and contributed to study design and discussion. EYC contributed data analysis and involved in revising the manuscript. MCC is the general supervision of the research group, and made substantial contributions to design of the study. All authors read and approved the final manuscript.

\section{Acknowledgements}

This work was supported by the Korea National Institute of Health intramural research grant, 4861-303-210-13 (2012-N63001-00, 2011-E63009-00).

\section{Author details}

${ }^{1}$ Division of Cardiovascular and Rare Diseases, Center for Biomedical Science, National Institute of Health, Chungbuk, Korea. ${ }^{2}$ Division of Cardiology, Department of Internal Medicine, Seoul National University Bundang Hospital, Bundang, Korea. ${ }^{3}$ Korean Nurses Association, Seoul, Korea. ${ }^{4}$ Channing Division of Network Medicine, Brigham and Women's Hospital and Harvard Medical School, Boston, MA, USA. ${ }^{5}$ Korea National Institute of Health, Chungbuk, Korea. ${ }^{6}$ Department of Internal Medicine, College of Medicine, Chungbuk National University, Chungbuk, Korea.

Received: 2 September 2013 Accepted: 16 December 2013 Published: 20 December 2013

\section{References}

1. Wang XS, Armstrong ME, Cairns BJ, Key TJ, Travis RC: Shift work and chronic disease: the epidemiological evidence. Occup Med (Lond) 2011, 61:78-89.

2. Bøggild $H$, Knutsson A: Shift work, risk factors and cardiovascular disease. Scand J Work Environ Health 1999, 25:85-99.

3. Knutsson A: Health disorders of shift workers. Occup Med (Lond) 2003, 53:103-108.

4. Antunes LC, Levandovski R, Dantas G, Caumo W, Hidalgo MP: Obesity and shift work: chronobiological aspects. Nutr Res Rev 2010, 23:155-168.

5. Di Lorenzo L, De Pergola G, Zocchetti C, L'Abbate N, Basso A, Pannacciulli N, Cignarelli M, Giorgino R, Soleo L: Effect of shift work on body mass index: results of a study performed in 319 glucose-tolerant men working in a Southern Italian industry. Int J Obes Relat Metab Disord 2003, 27:1353-1358.

6. Suwazono Y, Dochi M, Sakata K, Okubo Y, Oishi M, Tanaka K, Kobayashi E, Kido T, Nogawa K: A longitudinal study on the effect of shift work on weight gain in male Japanese workers. Obesity (Silver Spring) 2008, 16:1887-1893

7. Macagnan J, Pattussi MP, Canuto R, Henn RL, Fassa AG, Olinto MT: Impact of nightshift work on overweight and abdominal obesity among workers of a poultry processing plant in southern Brazil. Chronobiol Int 2012, 29:336-343.

8. van Drongelen A, Boot CR, Merkus SL, Smid T, van der Beek AJ: The effects of shift work on body weight change - a systematic review of longitudinal studies. Scand J Work Environ Health 2011, 37:263-275.

9. Buss J: Associations between obesity and stress and shift work among nurses. Workplace Health Saf 2012, 60:453-458.

10. Han K, Trinkoff AM, Storr CL, Geiger-Brown J: Job stress and work schedules in relation to nurse obesity. J Nurs Adm 2011, 41:488-495.

11. Zapka JM, Lemon SC, Magner RP, Hale J: Lifestyle behaviours and weight among hospital-based nurses. J Nurs Manag 2009, 17:853-860.

12. Flegal KM, Carroll MD, Kit BK, Ogden CL: Prevalence of obesity and trends in the distribution of body mass index among US adults, 1999-2010. JAMA 2012, 307:491-497.

13. Geliebter A, Gluck ME, Tanowitz M, Aronoff NJ, Zammit GK: Work-shift period and weight change. Nutrition 2000, 16:27-29.

14. van Amelsvoort LG, Schouten EG, Kok FJ: Duration of shiftwork related to body mass index and waist to hip ratio. Int J Obes Relat Metab Disord 1999, 23:973-978.

15. Parkes KR: Shift work and age as interactive predictors of body mass index among offshore workers. Scand J Work Environ Health 2002, 28:64-71.

16. Chee HL, Kandiah M, Khalid M, Shamsuddin K, Jamaluddin J, Nordin NA, Shuib R, Osman I: Body mass index and factors related to overweight among women workers in electronic factories in Peninsular Malaysia. Asia Pac J Clin Nutr 2004, 13:248-254.

17. Karlsson B, Knutsson A, Lindahl B: Is there an association between shift work and having a metabolic syndrome? Results from a population based study of 27,485 people. Occup Environ Med 2001, 58:747-752.

18. Rhee SY, Park SW, Kim DJ, Woo J: Gender disparity in the secular trends for obesity prevalence in Korea: analyses based on the KNHANES 1998-2009. Korean J Intern Med 2013, 28:29-34

19. Ishizaki M, Morikawa Y, Nakagawa H, Honda R, Kawakami N, Haratani T, Kobayashi F, Araki S, Yamada Y: The influence of work characteristics on body mass index and waist to hip ratio in Japanese employees. Ind Health 2004, 42:41-49.

20. Zhao I, Bogossian F, Song S, Turner C: The association between shift work and unhealthy weight: a cross-sectional analysis from the Nurses and Midwives' e-cohort Study. J Occup Environ Med 2011, 53:153-158.

21. Zhao I, Bogossian F, Turner C: A cross-sectional analysis of the association between night-only or rotating shift work and overweight/obesity among female nurses and midwives. J Occup Environ Med 2012, 54:834-840.

22. Persson M, Mårtensson J: Situations influencing habits in diet and exercise among nurses working night shift. J Nurs Manag 2006, 14:414-423.

23. Miller SK, Alpert PT, Cross CL: Overweight and obesity in nurses, advanced practice nurses, and nurse educators. J Am Acad Nurse Pract 2008 20:259-265.

doi:10.1186/1471-2458-13-1204

Cite this article as: Kim et al:: Association between shift work and obesity among female nurses: Korean Nurses' Survey. BMC Public Health 2013 13:1204.

\section{Submit your next manuscript to BioMed Central and take full advantage of:}

- Convenient online submission

- Thorough peer review

- No space constraints or color figure charges

- Immediate publication on acceptance

- Inclusion in PubMed, CAS, Scopus and Google Scholar

- Research which is freely available for redistribution 